\section{Nachhaltiger Finanzsektor?}

\section{Der Finanzdienstleistungssektor fand in der Nachhaltigkeitsdiskussion bisher wenig Beachfung, obwohl das fünfte Umweltaktionsprogramm der EU schon vor Jahren auf diesen Bereich hingewiesen hat. Wie sieht die mögliche Rolle von Banken und Versicherungen aus?}

\section{W}

Von Walter Kablenborn und

R. Andreas Kraemer

geht, sind Finanzdienstleister als Akteure in vielfältiger Weise angesprochen.

- Als Investoren beschaffen sie das notwendige Kapital für eine nachhhaltige oder nichtnachhaltige Entwicklung

- Als Berater wirken sie auf die Kunden und deren Verhalten ein.

- Als Bewerter kalkulieren sie Umweltrisiken und machen sie damit sichtbar.

- Als Einflußträger können sie die Unternehmenspolitik einiger ihrer Kunden, aber auch das Verhalten staatlicher Stellen erheblich beeinflussen.

- Als Verursacher von Umweltverschmutzung sind sie für direkte Umweltbelastungen verantwortlich, wenn auch weit weniger als andere Industriezweige.

- Als Opfer von Umweltverschmutzung müssen sie die entstandenen Schäden bewältigen.

Bisher geht der Sektor der Finanzdienstleister in Europa in der Praxis mit seiner Verantwortung recht unterschiedlich um. Der Sektor der Geschäftsbanken etwa konzentriert sich derzeit mit seinen Aktivitäten vor allem auf zwei Gebiete. Zum einen haben viele der Unternehmen erhebliche Fortschritte gemacht bei der Entwicklung interner Umweltmanagementsysteme, um die Umweltbelastungen aus ihren Betriebsabläufen zu reduzieren. Zum anderen beziehen die meisten Banken Informationen zum Umweltverhalten ihrer Kunden in die Kreditwürdigkeitsprüfung ein, wobei der ausschlaggebende Blickwinkel aber alleine die Haftungsrisiken sind.

Erst wenige Banken haben inzwischen ihre umweltbezogenen Aktivitäten ausgedehnt und stellen etwa umfangreiche Checklisten für die Kreditwürdigeitsprüfung auf oder bieten kleinen und mittleren Unternehmen besondere Unterstiitzung beim Aufbau eines effektiven Umweltmanagements. Kaum Ansätze bestehen bei der
Entwicklung neuer Finanzprodukte, die Umweltaspekte besonders berücksichtigen, wie beispielsweise spezielle Kredite für Energiesparmaßnahmen.

Beim Blick auf das Investmentgeschäft besteht noch allgemein eine gewisse Zurückhaltung. Ein wichtiger Grund hierfür ist der Zweifel vieler Investoren, daß ein aktiver betrieblicher Umweltschutz auch die finanzielle Rentabilität eines Unternehmens positiv beeinflußt. Hierbei kommt auch zum tragen, daß der Investmentsektor Schwierigkeiten hat, qualitativ hochwertige und gut aufbereitete Informationen zu erhalten, mittels derer die Umweltauswirkungen der Unternehmensaktivitäten sowie die damit verbundenen finanziellen Risiken schnell und einfach abgeschätzt werden können.

Insgesamt läßt sich in der Praxis derzeit feststellen, daß vor allem private Investoren nach ökologische Investitionsmöglichkeiten suchen. $\mathrm{Zu}$ diesem Zweck hat sich in der Zwischenzeit insbesondere der Nischenmarkt der Ökofonds und ökologisch motivierten Direktinvestitionen etabliert. Während hier zunächst nur in den angelsächsischen Ländern ein nennenswertes Potential bestand, dehnt sich der Markt jetzt auch in Deutschland immer stärker aus. Noch ist das Investitionsvolumen im Vergleich zum Gesamtmarkt aber sehr gering, und bei den großen institutionellen Anlegern finden sich erst sehr wenige Ansätze für eine ökologische Anlagepolitik.

\section{Zurückhaltung der Versicherungen}

Der dritte Bereich des Finanzsektors, die Versicherungen, verhält sich, bis auf den Klimaschutz, mit Blick auf nachhaltige Entwicklung zurückhaltend. Das betrifft sowohl die Entwicklung neuer „Öko"-Versicherungsprodukte als auch die Investitionspolitik, also die Frage, wo die erheblichen Finanzmittel angelegt werden. Stärker diskutiert wird derzeit auch, ob die Versicherungswirtschaft Kontrollaufgaben bezïglich der Einhaltung von Umweltvorschriften übernehmen könnte, indem strengere Haftungs- vorschriften mit einer Versicherungspflicht verbunden würden. Die Erfahrung in den Vereinigten Staaten hat jedoch gezeigt, daß dies im wesentlichen die Mitwirkung der Versicherungswirtschaft voraussetzt und deren Interesse an der Übernahme unbekannter und nur schwer abschätzbarer Versicherungsrisiken gering ist. Betrachtet man nach dieser recht groben Skizze der momentanen Situation die Frage, wie in Zukunft die Finanzdienstleister innerhalb der europäischen Union stärker in eine Entwicklung hin zur Nachhaltigkeit einbezogen werden können, dann ergeben sich eine Reihe von Ansatzpunkten. Einige davon sind:

- Ein verbesserter Fluß von Umweltinformationen, etwa durch die Entwicklung von Umweltberichtsstandards, die auf die Bedürfnisse der Finanzmärkte abgestellt sind.

- Die Verbreitung von Informationen zu best practice im Bereich der Finanzdienstleistung.

- Die Erweiterung der Öko-Audit-Verordnung der EU, so daß alle Finanzdienstleister eingeschlossen werden. Dabei käme es allerdings darauf an, stärker die Produktpolitik und weniger den betrieblichen Umweltschutz (Papierverbrauch etc.) zu berücksichtigen.

- Die Übernahme einer Vorreiterrolle durch die öffentlichen Finanzdienstleister.

- Die Entwicklung eines Öko-Labels für Finanzmarktprodukte, um einerseits ,grünen“ Produkten mehr Publizität $z u$ verschaffen und andererseits Verbrauchertäuschung zu verhindern.

\section{Anmerkung}

Weitere Vorschläge und eine umfongreiche Würdigung der Rolle der Finanzinstitutionen ouf dem Weg zu einer nachhaltigen Entwicklung enthält ein Bericht, den Ecologic zusammen mit Delphi International im Auftrog der Generaldirektion XI (Umwelt) der Europäischen Union erstellt hat. Der Bericht ist erhältlich in englischer Sproche bei der Information Unit der GD XI, Rue de la Loi 200, B - 1049 Brüssel, Tel. + 322 2968770; z.zt. noch kostenfrei.

\section{Die Autoren}

R. Andreas Kraemer ist einer der Geschäftsführer von Ecologic. Walter Kahlenborn ist als freier Mitarbeiter für "Ecologic" tätig. Beide arbeiten an verschiedenen internationalen und europäischen Projekten im Bereich "green finance" mit.

Kontakt: Ecologic, Friedrichstraße 165, $10117 \mathrm{Ber}$ lin, Tel. 030/22 6511 35, Fax 030/22 6511 36, E-mail: office@ecologic.de 
(c) 20I0 Authors; licensee IÖW and oekom verlag. This is an article distributed under the terms of the Creative Commons Attribution Non-Commercial No Derivates License (http://creativecommons.org/licenses/by-nc-nd/3.o/), which permits unrestricted use, distribution, and reproduction in any medium, provided the original work is properly cited. 